\title{
High Energy Physics Data Popularity : ATLAS Datasets Popularity Case Study
}

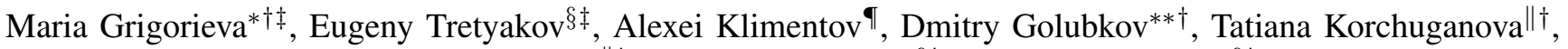 \\ Aleksandr Alekseev ${ }^{\| \dagger}$, Alexey Artamonov ${ }^{\S \dagger}$ and Timofei Galkin ${ }^{\S \ddagger}$ \\ ${ }^{*}$ Lomonosov Moscow State University, Moscow, Russian Federation \\ †Plekhanov Russian University of Economics, Moscow, Russian Federation \\ ${ }_{\ddagger}^{\ddagger}$ Moscow Center of Fundamental and Applied Mathematics, Moscow, Russian Federation \\ $\S$ National Research Nuclear University "MEPhI", Moscow, Russian Federation \\ "Brookhaven National Laboratory, Upton NY, USA \\ "Universidad Andrés Bello,Santiago, Chile \\ ** Institute for High Energy Physics of NRC Kurchatov Institute, Moscow, Russian Federation
}

\begin{abstract}
The amount of scientific data generated by the LHC experiments has hit the exabyte scale. These data are transferred, processed and analyzed in hundreds of computing centers. The popularity of data among individual physicists and University groups has become one of the key factors of efficient data management and processing. It was actively used during LHC Run 1 and Run 2 by the experiments for the central data processing, and allowed the optimization of data placement policies and to spread the workload more evenly over the existing computing resources. Besides the central data processing, the LHC experiments provide storage and computing resources for physics analysis to thousands of users. Taking into account the significant increase of data volume and processing time after the collider upgrade for the High Luminosity Runs (20272036) an intelligent data placement based on data access pattern becomes even more crucial than at the beginning of LHC. In this study we provide a detailed exploration of data popularity using ATLAS data samples. In addition, we analyze the geolocations of computing sites where the data were processed, and the locality of the home institutes of users carrying out physics analysis. Cartography visualization, based on this data, allows the correlation of existing data placement with physics needs, providing a better understanding of data utilization by different categories of user's tasks.

Index Terms-data popularity, ATLAS, LHC, data processing
\end{abstract}

\section{INTRODUCTION}

The ATLAS experiment [1] at the LHC utilizes distributed resources involving about 160 computing centers spread around the world for analysis, data simulation and processing. The ATLAS distributed data management system (Rucio) exploits special analytical algorithms ensuring the uniform distribution of the data over the existing computing sites. A dataset (collection of files processed using the same version of software) is a unit of data replication and processing in ATLAS. The dataset popularity is a metric showing how often a dataset was used as an input for physics analysis within a certain time interval. In this paper we discuss the existing approaches of the data popularity evaluation, and demonstrate the extension of the provided methods to the popularity of ATLAS datasets used for individual physics analysis.

\section{Data Processing in ATLAS}

Data processing in HEP can be represented as a sequence of steps to transform detector data to the formats applicable for physics analysis. A collection of processing jobs (organized as a computational task) are executed at each step. Usually task input and output(s) are datasets in different formats. A processing chain for detector data is relatively simple, having two main steps: reconstruction and derivation production.

- Detector data (RAW format) $\rightarrow$ Reconstruction step (produces AOD format) $^{1} \rightarrow$ Derivation step (produces $^{2}$ DAOD format $)^{2}$

For Monte Carlo simulation the above chain has extra steps related to event simulation and digitization. The above steps are orchestrated by the central production. In contrast to reconstruction and derivation production, physics analysis is not a centrally organized activity. Over one and a half thousand physicists in ATLAS submit more than ten millions tasks per year. Figure 1 demonstrates that the overall number of user analysis jobs completed in one month (highlighted with the green color) exceeds all other types of workflows ${ }^{3}$.

As the volume of data is rapidly growing, optimization of the storage capacity and computing resource utilization become a critical issue for the HL-LHC Run (aka Run 4). Thus, data popularity measurement and prediction are becoming an important part of analytical studies ensuring efficient and stable operation of the WLCG ${ }^{4}$ computing infrastructure.

The main difference between user analysis and central production activities is the manageability and predictability. The central production workflows are planned in advance and organized by the experiment and physics groups; user analysis workflows are more chaotic because bursts of task submissions might appear and there is also a correlation with major scientific events such as the main international conferences. The number of central production tasks and resources required

\footnotetext{
${ }^{1}$ Analysis Object Data

${ }^{2}$ Derived Analysis Object Data

${ }^{3}$ Physics analysis tasks

${ }^{4}$ Worldwide LHC Computing Grid
} 


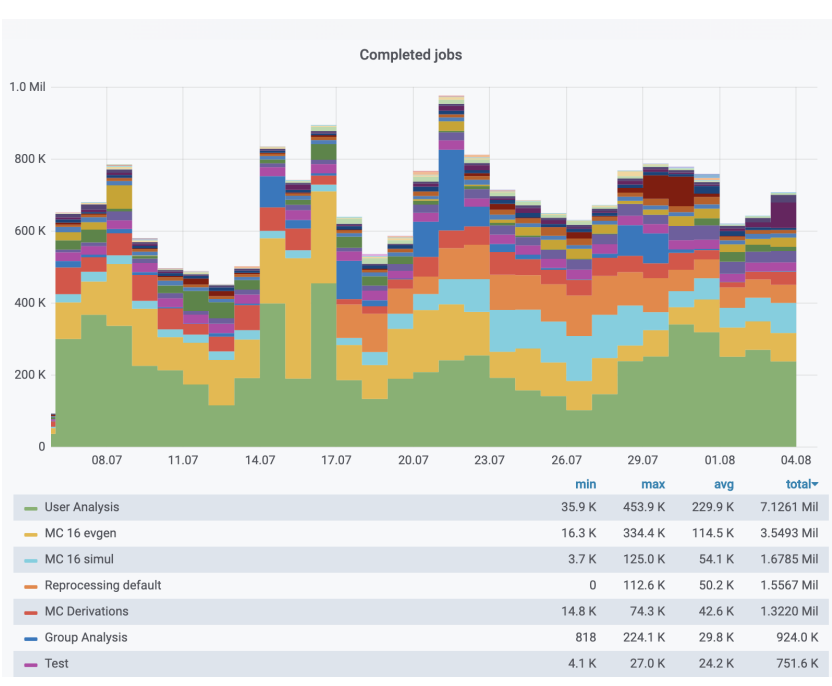

Fig. 1. Distribution of the completed jobs number by gShare (name of a group of physics analysis jobs).

could be estimated in advance, but the resources needed for users analysis can not be foreseen easily. That is why we devoted this research to the development of an analytical system to study popularity of the official ATLAS datasets exploited for individual user analysis tasks.

\section{Existing ApPROACHES OF DATA POPUlarity MEASUREMENTS IN ATLAS}

Two core systems are used for distributed data handling and processing in ATLAS:

- Distributed Data Management (DDM) Rucio [2] - keeps a catalogue of all ATLAS data on the WLCG sites; provides the organisation, transfer and management of ATLAS data.

- Workload Management System (WMS) PanDA [3] responsible for scheduling jobs on the grid based on available resources.

There were several attempts to have a stable and consistent data popularity services in LHC experiments [4] [5]. These services aggregate metadata about data transfers on a daily basis and calculate how many times a dataset was requested on the grid. The aim was to replicate the most popular datasets to optimize resources utilization, taking into account the popularity, locality, usage patterns of the data and available resources.

A Dynamic Data Placement Agent for ATLAS Distributed Data Management (C3PO) project [6] combines transfers, data accesses and data placement with network metrics, and dynamically creates new replicas, helping to spread the workload more evenly over existing computing resources. However, as it does not have tight integration with the WMS PanDA, and does not take into account the status of the computing queues, it is not used in central production workflows.

\section{ATLAS DATASETS POPULARITY FOR PHYSICS} ANALYSIS

When we deal with the individual user analysis based on the official ATLAS datasets, the popularity measured as the number of data transfers or accesses might not be enough. It would be interesting to evaluate the popularity of a dataset among universities, physics groups, regions and countries. For example, a dataset, accessed by users from several universities in different regions may be considered more popular than a dataset accessed by users from the same university.

Moreover, users sometimes download ATLAS datasets to carry out physics analysis on their local resources and such activities should also be taken into account as a popularity metric.

To meet these challenges we decided to design a new system, that is designed to analyse the popularity of ATLAS datasets used only for user analysis. We integrated metainformation about datasets, user analysis tasks and jobs, geolocations of users home institutes and computing sites with dataset replicas. All these parameters are collected and stored in ElasticSearch ${ }^{5}$ that provides flexible data queries and visualization with the help of Kibana ${ }^{6}$.

In the next sections we provide the description of data sources, used to collect needed information, and demonstrate the developed ETL workflow.

\section{DATA SOURCES}

Our main objective was to develop a stand-alone data base with data accumulated from different sources and aggregated in such a way that it provides the most convenient means to carry out popularity-specific queries and visualizations. Currently we use three data sources: PanDA Database, CRIC (Computing Resource Information Catalogue) and CERN LDAP.

\section{A. PanDA Database}

As the WMS PanDA supports both central production and individual user analysis, we can use the metadata from the database system serving PanDA - ATLAS_PANDA. It is based on Oracle RDBMS and includes multiple tables, storing the comprehensive static and dynamic information on all tasks, jobs and datasets in the system. To obtain the integral view of all ATLAS datasets, exploited in user analysis tasks, we joined the information from four data tables:

- ATLAS_PANDA.JEDI_DATASETS - metadata about datasets used in analysis tasks

- ATLAS_PANDA.JEDI_TASKS - provides the information about all tasks

- ATLAS_PANDA.JOBSARCHIVED4 - metadata about completed PanDA jobs for the previous 4 days

- ATLAS_PANDAARCH.JOBSARCHIVED - archived metadata about completed PanDA jobs for last 3 years

\footnotetext{
${ }^{5}$ https://www.elastic.co/elasticsearch/

${ }^{6} \mathrm{https} / / /$ www.elastic.co/kibana
} 
To construct the query we applied some filters that allowed us to limit the output to only user analysis tasks using official ATLAS data or Monte-Carlo datasets as an input. For the datetime ranges, we used granularity by a day, and for each day only completed tasks were collected.

The output data have the following parameters:

- Dataset name,

- gShare - name of the job flow-group (i.e. User Analysis, Group Analysis),

- User name - account or the name of user, initiated the physics analysis task,

- jeditaskid - ID of user analysis task,

- Creation date of a task (rounded by day),

- End date of a task (rounded by day),

- Status of a task (finished, failed, broken, cancelled, aborted, etc.),

- computing resource (single core or multi core),

- Panda queue,

- Size of input data (calculated as sum of input data for all relevant jobs),

- Size of output data (sum of output data for all relevant jobs).

Dataset name is composed of a set of decimal separated fields. Each field has a particular meaning relevant to the ATLAS Dataset Nomenclature document ${ }^{7}$.

Monte Carlo Datasets structure:

mcNN_subProject.datasetNumber.

physicshort.prodstep.dataType.Version

Real Data (Primary) Datasets structure:

DataNN_subProject.runNumber.

streamName.prodStep.dataType.Version

Dataset name is separated by a colon from the Rucio scope (scope:dataset name). The scope allows the separation of datasets produced by ATLAS central production from those created by individual groups or users. To ensure flexible searches by parameters encrypted in dataset names we split them by dots and store as the separate parameters:

- Project - the particular physics or computing context of a set of datasets,

- Rucio scope - for central production datasets, the scope is equivalent to the Project field value,

- Dataset number (processID) is in general associated with a unique physicsShort text comment,

- Run number - The DAQ run number,

- Physics short - text description of the event generation and detector simulation of the events in this dataset,

- Production step which was used to create the data (i.e. evgen, simul, recon, deriv),

- Data type (i.e. DAOD, $\mathrm{AOD}, \mathrm{ESD}^{8}, \mathrm{TAG}^{9}$ ),

${ }^{7}$ restricted to ATLAS members

${ }^{8}$ Event Summary Data

${ }^{9}$ Event Tags
- Stream name - the data stream, which is part of the filenames, and is assigned by the online Trigger, online $\mathrm{TDAQ}^{10}$ or offline reconstructed debug streams,

- AMI_tags (as Version) - AMI Tags ${ }^{11}$ are the last part of the dataset name. Tags represent successive production steps. They are concatenated to make the dataset configuration tag.

\section{B. $C R I C$}

CRIC describes the topology of the WLCG infrastructure (resource center sites, federations, storage services, experiment sites etc.) as well as experiment-specific configuration required to exploit this infrastructure [7]. In particular, CRIC provides APIs to obtain information about PanDA queues, ATLAS sites, storage systems, services and so on. We used the ATLAS Site API to identify the name of the site by the name of queue from a previous Oracle query, and some auxiliary parameters associated with the queues. Thus, the initial list of parameters were enhanced with the following:

- ATLAS site name,

- ATLAS site geolocation,

- Cloud name,

- Number of cores at site,

- Core Power of a site (result of HEP-SPEC06 ${ }^{12}$ benchmark),

- Tier level (i.e. Tier-1, Tier-2),

- Resource type (hpc_special, cloud, GRID, GPU,...).

Then the ATLAS Sites CRIC API was used to extract geolocations for the identified ATLAS sites.

\section{CERN Phonebook Directory}

The LDAP service ${ }^{13}$ allows anonymous read-access (from inside CERN only) to the user information in Active Directory. In particular, it contains the account information for all CERN users, and the membership of the CERN E-groups. We exploit this service to enhance our parameters with the title of the user's home institution and its country, and then apply a special service to find geolocations of the countries. Finally, the following parameters were added:

- User home institute,

- Home institute country,

- Country geolocation.

Figure 2 demonstrates schematically the whole data integration chain:

\section{EXTRACt TRANSForm LOAD (ETL) WORKFlOW}

It is convenient to describe the ETL workflow with Directed Acyclic Graphs (DAGs). The ETL DAG consists of nodes with directional dependencies. Each node is a run-unit that represents a staged part of a workflow. A DAG has a schedule, a start time and optional end time. The DAGs schedule can be

\footnotetext{
${ }^{10}$ ATLAS Trigger and Acquisition system

${ }^{11}$ AMI - ATLAS Metadata Interface

${ }^{12}$ https://w3.hepix.org/benchmarking/how_to_run_hs06.html

${ }^{13} \mathrm{https}: / /$ directory.web.cern.ch/
} 


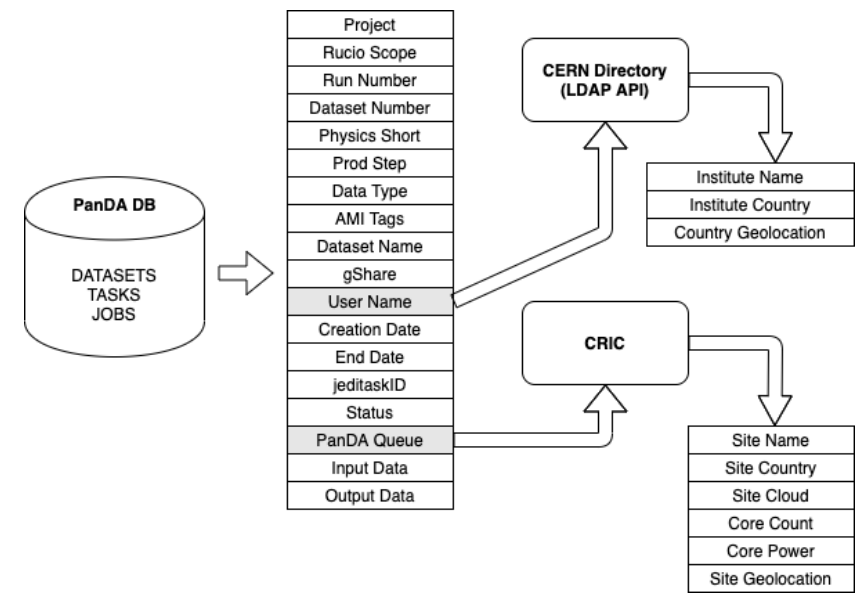

Fig. 2. Data Integration Structure.

hourly, each minute, etc. The scheduled DAG has to execute each individual run-unit as their dependencies are met.

The Apache Airflow ${ }^{14}$ open-source software is used to implement the ETL DAG approach. The Apache Airflow supports many databases, executors and message brokers. We decided to use Airflow based on PostgreSQL ${ }^{15}$ as backend database, Celery ${ }^{16}$ as the work executor and RabbitMQ ${ }^{17}$ as the message broker. Outside the Airflow environment, Elasticsearch and Kibana are used to store and analyse our data.

The data popularity ETL workflow to collect, merge and store data consists of two run-units with a communication feature between them. These run-units are "extract_data" and "push_to_elasticsearch". The "extract_data" run-unit collects metainformation from the data sources (PanDA Database, CRIC and CERN LDAP) and puts the relevant file into local storage. After that, the absolute path of the file is transferred to the next run-unit using XCOM. The Airflow XCOM stands for "cross-communication" and enables message exchange between run-units. As we have only one working node it is appropriate to use local storage but for scalability in the future some Content Delivery Network (CDN) will need to be used. The "push_to_elasticsearch" run-unit reads the file and bulks the data to elasticsearch. The complete ETL workflow is described in Figure 3.

\section{Popularity Measurements}

In this section we describe the popularity metrics that can be inferred from the collected data. The results presented cover datasets that were used in user analysis tasks during one month (July 2020).

The popularity measurements can be divided into four metrics:

- Tasks - the unique number of user analysis tasks that used datasets as an input. It refers to the number of accesses;

\footnotetext{
${ }^{14}$ https://airflow.apache.org/

${ }^{15} \mathrm{https} / / /$ www.postgresql.org/

${ }^{16} \mathrm{https}: / /$ docs.celeryproject.org/en/stable/

${ }^{17}$ https://www.rabbitmq.com/
}

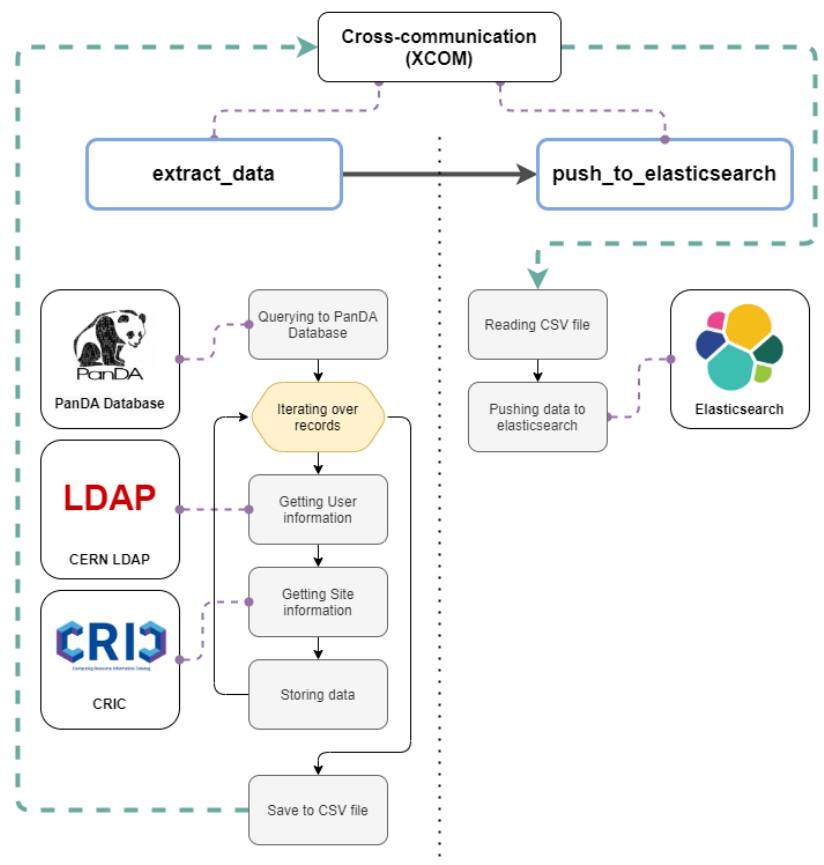

Fig. 3. ETL Workflow.

TABLE I

OVERall Popularity Metrics

\begin{tabular}{|c|c|}
\hline Metric & Value \\
\hline Tasks & 92,803 \\
\hline Users & 538 \\
\hline Institutes & 146 \\
\hline Countries & 34 \\
\hline
\end{tabular}

- Users - the unique number of users which executed analysis tasks;

- Institutes - the unique number of user home institutes;

- Countries - the unique number of home institute countries

In the period of 1-31 July 2020 the popularity of the official ATLAS datasets for user analysis was calculated as shown in Table I. However, not all of these datasets are really popular for user analysis. If we explore the statistical distribution of datasets by the number of tasks (Figure 4), it shows that the overwhelming majority of datasets are not popular (these datasets are visualized as the blue bar at the beginning of TASKS axis). Among almost $90 \mathrm{~K}$ datasets there are only 23 of them having number of accesses of more than 100 . We can conclude that in July there were only a few really popular datasets. The most popular of them is "mc15_13TeV:mc15_13TeV.410000.PowhegPythiaEvtGen _P2012_ttbar_hdamp172p5_nonallhad.recon.RDO. e3698_s2608_s2183_r7193_tid06752771_00”. It was exploited by 380 analysis tasks, carried out by two users from different universities (and countries).

The distribution of the number of datasets by the number 


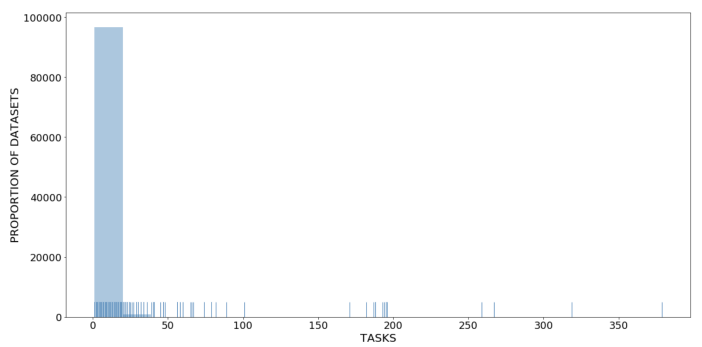

Fig. 4. Distribution of the popularity metrics by the number of tasks.

TABLE II

POPULARITY BY PROJECT

\begin{tabular}{|c|c|c|c|c|}
\hline Project & Tasks & Users & Institutes & Countries \\
\hline mc16_13TeV & 65,249 & 444 & 130 & 30 \\
\hline data18_13TeV & 7,833 & 153 & 86 & 24 \\
\hline data17_13TeV & 6,624 & 136 & 80 & 24 \\
\hline data16_13TeV & 5,803 & 124 & 73 & 22 \\
\hline data15_13TeV & 3,465 & 125 & 72 & 22 \\
\hline
\end{tabular}

of users (Figure 5) demonstrates that the most unpopular datasets are exploited by 1-5 users. And there are some datasets that were analyzed by $10-16$ users.

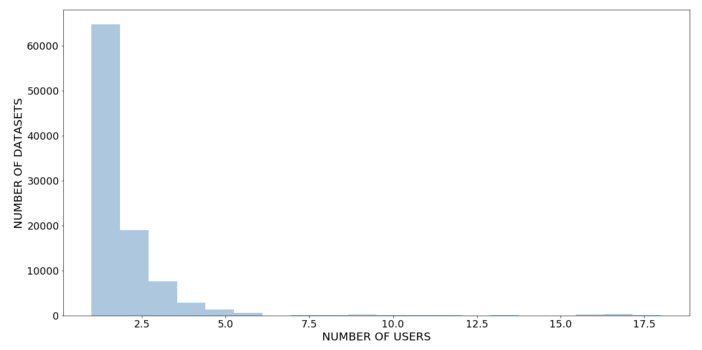

Fig. 5. Distribution of the popularity metrics by the number of users.

Table II demonstrates the popularity metrics of the top five ATLAS projects (the project name is encoded in dataset names, so as we store it as the parameter, it allows us to group dataset by projects): the most popular by the number of accesses (Tasks) and number of users are Monte-Carlo datasets of project mc16_13TeV. One of the most convenient ways to explore the popularity with multiple parameters is a parallel coordinates diagram, as shown in Figure 6. The diagram shows, graphically, three main groups of datasets: the first group mc16_13TeV is the most popular (blue bold curve); another group, that is visually below the first one, is less popular (i.e. data15_13TeV, data16_13TeV, data17_13TeV, data18_13TeV); and the third is least popular (i.e. data18_hi, mc16_5TeV, mc15_13TeV). The cartography visualization in Figure 7 and Table III show that this group of datasets (mc16_13TeV) is analyzed mainly in Europe (United Kingdom), United States, China and Canada.

We can go further and identify the universities involved in the analysis of mc16_13TeV data (as shown in Table IV).

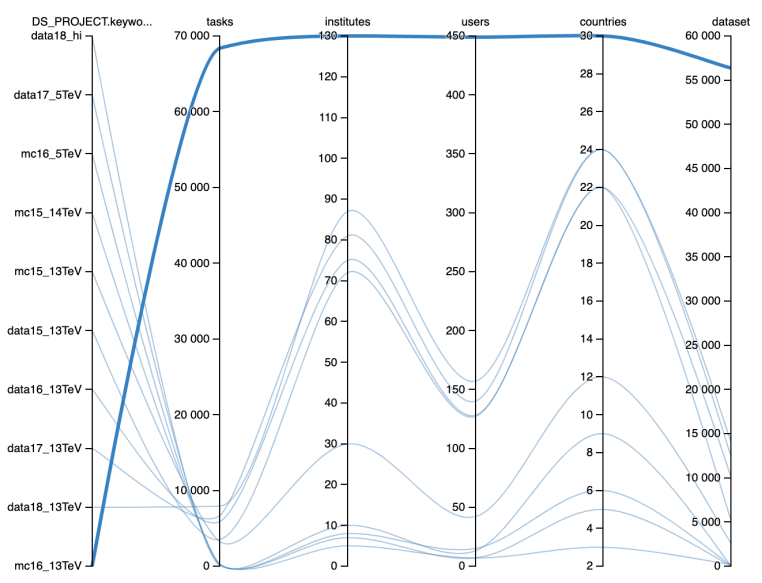

Fig. 6. Distribution of the popularity metrics by the projects - parallel coordinates diagram.

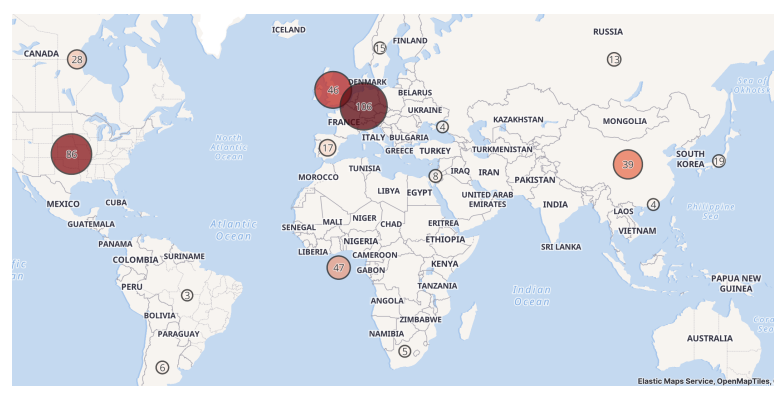

Fig. 7. Distribution of the popularity metrics by the number of users (mc16_13TeV).

TABLE III

POPULARITY BY COUNTRY

\begin{tabular}{|c|c|c|c|}
\hline Countries & Tasks & Users & Institutes \\
\hline UNITED STATES & 12,976 & 86 & 30 \\
\hline UNITED KINGDOM & 11,976 & 46 & 12 \\
\hline CHINA & 8,514 & 39 & 7 \\
\hline GERMANY & 8,490 & 63 & 13 \\
\hline CANADA & 3,516 & 28 & 8 \\
\hline
\end{tabular}

TABLE IV

POPUlARITY By USERS HOME INSTITUTES

\begin{tabular}{|c|c|c|}
\hline Institute & Tasks & Users \\
\hline University of Liverpool (GB) & 7,910 & 5 \\
\hline University of Michigan (US) & 1,086 & 7 \\
\hline University of Glasgow (GB) & 959 & 2 \\
\hline University of London (GB) & 723 & 7 \\
\hline University of Sussex (GB) & 473 & 2 \\
\hline
\end{tabular}

As we collect some information about tasks execution process, like computing sites with geolocations, we can visually explore where the analyzed datasets were processed. This will allow conclusions to be made about data placement policies (Figure 8). Another available option is to group popularity 


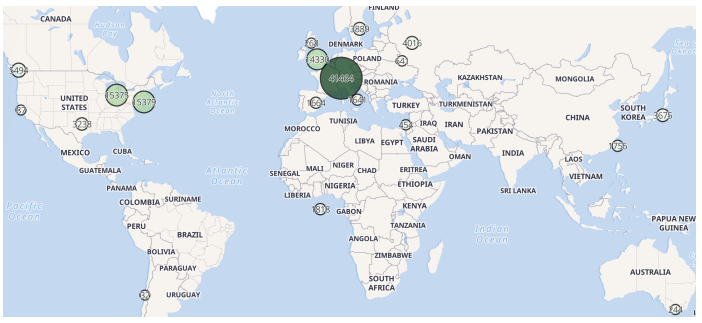

Fig. 8. Distribution of the popularity metrics by the number of tasks on computing sites (mc16_13TeV).

TABLE V

POPUlARITY By PRODUCTION STEP

\begin{tabular}{|c|c|c|c|c|}
\hline Production Step & Tasks & Users & Institutes & Countries \\
\hline deriv & 84,684 & 395 & 128 & 29 \\
\hline merge & 5,063 & 148 & 79 & 26 \\
\hline recon & 2,766 & 49 & 36 & 17 \\
\hline evgen & 258 & 23 & 20 & 8 \\
\hline digit & 84 & 1 & 1 & 1 \\
\hline
\end{tabular}

TABLE VI

POPUlARITY BY DATA TYPE

\begin{tabular}{|c|c|c|c|c|}
\hline Data Type & Tasks & Users & Institutes & Countries \\
\hline DAOD & 87,721 & 409 & 130 & 29 \\
\hline RDO & 2293 & 10 & 10 & 8 \\
\hline AOD & 1921 & 103 & 60 & 25 \\
\hline EVNT & 628 & 45 & 32 & 16 \\
\hline ESD & 90 & 13 & 10 & 7 \\
\hline
\end{tabular}

metrics by production steps of data processing. Table $\mathrm{V}$ shows that the most actively analyzed production step is derivation (deriv). All others - merge, reconstruction, event generation are statistically not significant.

The next table (Table VI) demonstrates distribution of the popularity metrics by data types. Here we can observe that users mostly analyse DAOD datasets (87K tasks and 492 users). AOD datasets are drastically less popular, but still in use for certain analyses: 103 users carried out $2 \mathrm{~K}$ analysis tasks with AOD data.

To calculate the overall popularity of a dataset or a group of datasets we propose the following formulas:

- normalize each popularity metric for all existing datasets or groups of datasets (i.e. projects, production steps)

$$
\frac{x-\min _{x}}{\max _{x}-\min _{x}}
$$

where $\mathrm{x}$ - is the value of a popularity metric in a group - calculate the popularity by the formula:

$$
\frac{N_{\text {tasks }}}{N_{\text {users }} / N_{\text {institutes }} / N_{\text {countries }}}
$$

Table VII shows the overall popularity calculated for each project in appliance with the data from Table II. In contrast to
TABLE VII

POPULARITY BY PROJECT

\begin{tabular}{|c|c|}
\hline Project & Popularity \\
\hline mc16_13TeV & 1.00 \\
\hline data18_13TeV & 0.23 \\
\hline data17_13TeV & 0.21 \\
\hline data16_13TeV & 0.17 \\
\hline data15_13TeV & 0.09 \\
\hline
\end{tabular}

the existing in ATLAS methods of the popularity evaluation, our approach involves four parameters that represent not only quantitative indicators of the popularity, but the variability of datasets utilization. The overall popularity metric takes into account all four measurements, providing more flexible and relevant values, than just the number of transfers or dataset accesses.

The correlations between the dataset popularity metric and the number of dataset replicas at a particular time frame will demonstrate the efficiency of the existing replication strategy. Thus, further development of the data popularity storage is tightly connected with the exploration and analysis of the DDM metadata.

Besides that, testing the overall popularity metric efficiency means building the accurate simulation models. WMS PanDA is a production system with a huge workload that has to be durable and sustainable. Thus, there is no ability to test every new approach related to data popularity usage efficiency on production system. Moreover a reasonable amount of data is needed to develop the popularity simulation model. To build this model we need to add more parameters. for example, the information about the number of datasets replicas and theirs locations.

\section{FUTURE WORK}

In contrast to canonical methods of popularity evaluation, which use the number of tasks created during a certain period, we used the number of completed tasks. This is because the full information set is only available for successfully completed tasks. This information includes the number of created jobs, computing queues/sites names where jobs were executed, processed and produced data volume, etc. The drawback of this approach is that in collecting data we do not take into account computing tasks that might have started and have not finished during the time window we are examining. These tasks will be collected with delay once they are completed. On the other hand, if we collect data by creation time of tasks, we don't record tasks that were started a couple of days before. So, in both cases, not all tasks are recorded. Statistics show that the numbers of created and completed tasks in a reasonable time period (more than a week) are statistically almost equal, however there might be fluctuations during one day. Statistics for May 2020 showed 102,358 created against 107,268 finalized tasks. Statistics for all June 2020: 92,728 created and 91,895 finished. It shows that the 
overall value of data popularity, of course, depends on the method of evaluation, but not critically. Collecting tasks that had finished to the particular day in future will help to analyse the resource utilization for popular tasks/datasets. However, as an improvement, we are going to implement two separate workflows - one to collect only newly created tasks, another to collect only finalized tasks. Then, these two views can be merged to avoid duplicates.

Not all geolocations of computing sites were found using CRIC API. Among 90K of analysis tasks we did not find geolocations for about $2 \mathrm{~K}$ of them.

Sometimes users specify non-standard usernames in task configurations (for example, users may add email addresses to the string with the username). In this case now we will not find the information about this user in the CERN Directory. But this issue can be solved partially by removing unnecessary emails from username parameter. Currently we did not find users locations for $8 \mathrm{~K}$ tasks.

The most important stage of further development is the addition of the information about the number of dataset replicas from Rucio API. Collection of this data with the Rucio API may be done only in an online mode. For the retrospective analysis we have to explore Rucio traces logs.

Additionally we plan to add one more data source DDM Rucio transfers to analyze which ATLAS datasets were downloaded by users to their local resources. The number of such downloads will become one more metric of datasets popularity.

Finally, the web-application will be designed aimed at the visual analysis of data popularity, with the application of advanced interactive visualization techniques like parallel coordinates, world maps, heatmaps and treemaps.

\section{CONCLUSiON}

This research is the starting point for the development of a data popularity system for ATLAS user analysis. It was created as a separate project because user analysis activities have some peculiarities that could be explored as separately from the central data analysis, i.e. unpredictable number of user analysis tasks under the conditions of growing data volumes in ATLAS. The system is expected to ensure fast and flexible access to all popularity metrics that are specific for user analysis, and to provide means to make decisions about the optimization of data replication policies or data distribution between cache, disk and tape storages. As we collect data on a daily basis, it enable additionally the exploratory analysis of the life-cycle of datasets to evaluate how data popularity changes in time.

\section{ACKNowledgements}

This work is supported by the Russian Science Foundation grant \#19-71-30008 in part related to the development and maintaining of popularity storage and HEP data samples popularity studies (this research is conducted in the Plekhanov Russian University of Economics), and by the Moscow Center of Fundamental and Applied Mathematics in part related to the implementation of ETL-workflows.

\section{REFERENCES}

[1] ATLAS Collaboration, The ATLAS Experiment at the CERN Large Hadron Collider, JINST 3 (2008) S08003

[2] M. Barisits et al. Rucio - Scientific data management, Comput. Softw. Big Sci. 3 (2019) 11

[3] F. H. Barreiro Megino et al. PanDA for ATLAS distributed computing in the next decade, J. Phys. Conf. Ser. 898 (2017) 052002

[4] T. Maeno et al. PD2P: PanDA Dynamic Data Placement for ATLAS, J. Phys. Conf. Ser. 396 (2012) 32070

[5] M. Hushchyn et al. Disk storage management for LHCb based on Data Popularity estimator, J. Phys. Conf. Ser. 664 (2015) 042026

[6] T. Beermann et al. C3PO - A dynamic data placement agent for ATLAS distributed data management, J. Phys. Conf. Ser. 898 (2017) 062012

[7] A. Anisenkov et al. CRIC: a unified information system for WLCG and beyond, EPJ Web Conf. 214 (2019) 03003 